\title{
Cereulide synthetase gene cluster from emetic Bacillus cereus: Structure and location on a mega virulence plasmid related to Bacillus anthracis toxin plasmid pXO I Monika Ehling-Schulz*1, Martina Fricker ${ }^{1}$, Harald Grallert ${ }^{1}$, Petra Rieck ${ }^{2}$, Martin Wagner ${ }^{2}$ and Siegfried Scherer ${ }^{1}$
}

Address: ${ }^{1}$ Lehrstuhl für Mikrobielle Ökologie, Department für Biowissenschaftliche Grundlagen, WZW, Technische Universität München, Freising, Germany and 2Institute of Milk Hygiene, Milk Technology and Food Science, University of Veterinary Medicine, Vienna, Austria

Email: Monika Ehling-Schulz* - Monika.Ehling-Schulz@wzw.tum.de; Martina Fricker - Martina.Fricker@wzw.tum.de;

Harald Grallert - Harald.Grallert@gsf.de; Petra Rieck - Petra.Rieck@vu-wien.ac.at; Martin Wagner - Martin.Wagner@vu-wien.ac.at; Siegfried Scherer - Siegfried.Scherer@wzw.tum.de

* Corresponding author

Published: 02 March 2006

BMC Microbiology2006, 6:20 doi:10.1/86/147|-2180-6-20
Received: 30 November 2005

Accepted: 02 March 2006

This article is available from: http://www.biomedcentral.com/I47I-2/80/6/20

(C) 2006Ehling-Schulz et al; licensee BioMed Central Ltd.

This is an Open Access article distributed under the terms of the Creative Commons Attribution License (http://creativecommons.org/licenses/by/2.0), which permits unrestricted use, distribution, and reproduction in any medium, provided the original work is properly cited.

\begin{abstract}
Background: Cereulide, a depsipeptide structurally related to valinomycin, is responsible for the emetic type of gastrointestinal disease caused by Bacillus cereus. Recently, it has been shown that this toxin is produced by a nonribosomal peptide synthetase (NRPS), but its exact genetic organization and biochemical synthesis is unknown.

Results: The complete sequence of the cereulide synthetase (ces) gene cluster, which encodes the enzymatic machinery required for the biosynthesis of cereulide, was dissected. The $24 \mathrm{~kb}$ ces gene cluster comprises 7 CDSs and includes, besides the typical NRPS genes like a phosphopantetheinyl transferase and two CDSs encoding enzyme modules for the activation and incorporation of monomers in the growing peptide chain, a CDS encoding a putative hydrolase in the upstream region and an $A B C$ transporter in the downstream part. The enzyme modules responsible for incorporation of the hydroxyl acids showed an unusual structure while the modules responsible for the activation of the amino acids Ala and Val showed the typical domain organization of NRPS. The ces gene locus is flanked by genetic regions with high homology to virulence plasmids of $B$. cereus, Bacillus thuringiensis and Bacillus anthracis. PFGE and Southern hybridization showed that the ces genes are restricted to emetic $B$. cereus and indeed located on a $208 \mathrm{~kb}$ megaplasmid, which has high similarities to $\mathrm{pXOI-like} \mathrm{plasmids.}$

Conclusion: The ces gene cluster that is located on a PXOI-like virulence plasmid represents, beside the insecticidal and the anthrax toxins, a third type of $B$. cereus group toxins encoded on megaplasmids. The ces genes are restricted to emetic toxin producers, but $\mathrm{pXOI}$-like plasmids are also present in emetic-like strains. These data might indicate the presence of an ancient plasmid in $B$. cereus which has acquired different virulence genes over time. Due to the unusual structure of the hydroxyl acid incorporating enzyme modules of Ces, substantial biochemical efforts will be required to dissect the complete biochemical pathway of cereulide synthesis.
\end{abstract}




\section{Background}

Bacillus cereus belongs to the B. cereus group of organisms that comprises the genetically highly related organisms Bacillus anthracis, B. cereus, Bacillus mycoides, Bacillus pseudomycoides, Bacillus thuringiensis, and Bacillus weihenstephanensis, $(16,33)$ which for the most part are capable of producing significantly different toxins. B. anthracis causes the fatal animal and human disease anthrax, whereas $B$. thuringiensis produces insecticidal toxins that are commercially used as biocontrol agents [1-3]. Toxin producing $B$. cereus plays an important role as the causative agent of two types of food poisoning: diarrhea and emesis (for review see Granum [4] and Ehling-Schulz et al., [5]). The emetic syndrome caused by B. cereus is mainly characterized by vomiting a few hours after ingestion of the contaminated food while diarrhoeal poisoning is caused by heat-labile enterotoxins produced during vegetative growth of $B$. cereus in the small intestine [6]. The latter, diarrhea eliciting, toxins are well characterized at the molecular and the transcriptional level [7-10]. Far less is known about the emesis causing toxin cereulide, which has twice been reported to have been involved in the death of a child due to liver failure $[11,12]$.

Cereulide is a small, heat and acid stable cyclic dodecadepsipeptide which is chemically closely related to the potassium ionophore valinomycin [13]. It is toxic to mitochondria by acting as a potassium ionophore (2, 25)and has been reported to inhibit human natural killer cells [14]. According to its chemical structure one could expect cereulide to be synthesized enzymatically via nonribosomal peptide synthetases (NRPSs).

NRPSs are large multifunctional proteins that have a modular organization. The modules, which show a conserved domain structure, selectively catalyze activation and thioester formation of one amino, $\alpha$-hydroxy or carboxylic acid monomer $[15,16]$. A minimal module comprises an adenylation (A domain) that activates the cognate substrate and a thiolation domain (T domain) that binds the activated substrate. Chain elongation of the peptide is catalyzed by condensation domains ( $\mathrm{C}$ domains), located at $\mathrm{N}$-terminal ends of modules which accept acyl groups from the preceding modules [17]. A C-terminal thioesterase domain (TE domain) catalyzes the release of the mature NRPS-bound peptide product [18]. Some modules contain additional domains, like epimerization and methylation domains, that modify the incorporated constituents (for review see Sieber and Marahiel [19]). The order of modules usually corresponds directly to the order of monomers in the peptide product [20,21]. Quite recently, we identified a putative valine activating module that was highly conserved among emetic B. cereus. Disruption of the corresponding gene by insertion mutagenesis revealed cereulide deficient mutants, providing for the first time unequivocal evidence for nonribosomal assembly of the emetic toxin cereulide [22].

The main virulence factors of $B$. anthracis and insecticidal toxin genes of $B$. thuringiensis are located on large, well characterized virulence plasmids while diarrhea eliciting enterotoxins of $B$. cereus are located chromosomally. However, the exact genomic location and organization of the cereulide synthetase genes, which represent the main virulence factors of the emetic type of $B$. cereus, was hitherto unknown. The aim of this work was to completely sequence and characterize the genetic locus responsible for cereulide synthesis including the flanking genomic regions in order to gain insight into the synthesis mechanism of this peptide toxin, to determine the genomic location of the ces gene cluster and to study its conservation among $B$. cereus group members.

\section{Results}

Organization of the cereulide synthetase (ces) gene cluster The total sequence of the cereulide synthetase gene cluster (24 kb) was dissected by module jumping and inverse PCR. Sequence analysis showed that the ces gene cluster encodes 7 CDSs: cesH, cesP, cesT, ces A, ces B, ces $C$ and cesD (Fig. 1). CesH is located at the 5' end of the cluster $990 \mathrm{bp}$ upstream of cesP in the same reading frame. cesT is located 268 bp downstream from cesP while cesA overlaps with cesT. ces $B$ is separated by 13 bp from the stop codon of ces $A$. ces $C$ and ces $D$ are located in the 3 ' part of the ces gene cluster (Fig. 1). No termination structures were predicted, neither between the CDSs cesPTABCD nor between cesH and $c e s P$, but downstream of the ces $D$ gene a hairpin structure was predicted using Mfold [23], indicative of the presence of a terminator sequence. The deduced protein sequence (Table 2) from cesH (31 kDa) showed significant (58\%) identity to putative hydrolases/acyltransferases (COG0596) from $B$. cereus group members. The following CDS, designated cesP (28.9 kDa), showed high homology (32-38\% identity and approx. 60\% similarity) to the 4'-phosphopantetheinyl transferase from Bacillus brevis and Bacillus subtilis involved in nonribosomal synthesis of gramicidin S (Gsp; Swissprot database accession no. P40683) and to surfactin (Sfp; Swissprot database accession no. P39135), respectively [24,25]. cesT starts with a GTG and is preceded by a putative RBS (AGGAG) 5 bp upstream of the start codon. CesT (27.6 kDa) showed significant similarity to BacT from Bacillus licheniformis (GenBank accession no. AF007865.2; 33\% identity and $56 \%$ similarity), GrsT from B. brevis (GenBank accession no. AJ566197; 35\% identity, 53\% similarity) and other thioesterases associated with NRPS. The structural genes of cereulide synthetase are coded by the two large CDSs ( $10 \mathrm{~kb}$ and $8 \mathrm{~kb}$ ) ces $A$ and $\operatorname{ces} B$. CesA and CesB, each of which is responsible for the activation and incorporation of two monomers in the peptide chain, show high homol- 
Table I: Oligonucleotide primers used for module jumping and amplifications

\begin{tabular}{|c|c|c|}
\hline Primer & Sequence (5'-3') & Use \\
\hline F_C3a & $\begin{array}{l}\text { GCA(CT)CA(CT)AT(ACT)AT(ACT)TC(AGCT)GA(CT)GG(AGCT } \\
) \text { TGG }\end{array}$ & Module jumping (C-A domain) \\
\hline R_TIa & $\mathrm{C}(\mathrm{AGT}) \mathrm{A}(\mathrm{GT})(\mathrm{AGT}) \mathrm{A}(\mathrm{AG})(\mathrm{AT}) \mathrm{GA}(\mathrm{AG}) \mathrm{TG}(\mathrm{ACT}) \mathrm{CC}(\mathrm{AC}) \mathrm{CC}$ & Module jumping (A-T domain) \\
\hline F_A3 & $\begin{array}{l}\mathrm{GG}(\mathrm{ACT})(\mathrm{AT}) \mathrm{C}(\mathrm{AGT}) \mathrm{AC}(\mathrm{ACT}) \mathrm{GG}(\mathrm{ACT})(\mathrm{AC}) \mathrm{A}(\mathrm{AGCT}) \mathrm{CC}(\mathrm{ACT}) \\
\mathrm{AA}(\mathrm{AG}) \mathrm{GG}\end{array}$ & Module jumping (A-T domain) \\
\hline PLI0987_R2 & CCGTTATCAGCAATAGTCCTA & Primer derived from $\mathrm{pBc} 10987$ \\
\hline PLI0987_R4 & GAATATACTCAACAGTAGCTACC & Primer derived from $\mathrm{pBcl} 0987$ \\
\hline PLI0987_R5 & CGATACGTCAAATGTACTCGG & Primer derived from $\mathrm{pBcl} 0987$ \\
\hline PLI0987_R6 & GTGATTCATATTGTCTGGATACG & Primer derived from $\mathrm{pBc} 10987$ \\
\hline PLI0987_F20 & GAAGAGAGATATGTCTGTACT & Primer derived from $\mathrm{pBc} 10987$ \\
\hline PLI0987_F2I & GTATATTGTGGCAATTGATGAAGC & Primer derived from $\mathrm{pBc} 10987$ \\
\hline
\end{tabular}

a) see reference [22]. Primers for hybridization studies are provided in the supplement (see additional file Table SI); primers for inverse PCR and control sequence reaction are not shown

ogy to known NRPS and PKS-NRPS hybrids. Alignment of CesA and CesB revealed 32\% identity and 53\% similarity between these two proteins. ces $C$ and cesD, located in the 3 ' part of the ces gene cluster, encode a putative $\mathrm{ABC}$ transporter. $\mathrm{ABC}$ binding cassettes and transmembrane domains, typical for the $\mathrm{ABC}$ transporter family, were identified in these sequences.

\section{Analysis of the NRPS domains of cereulide synthetases} The CesA synthetase harbors two modules with the domain structure $\mathrm{A}_{\mathrm{i}}-\mathrm{X}-\mathrm{A}_{\mathrm{ii}}-\mathrm{T}-\mathrm{C}-\mathrm{A}-\mathrm{T}-\mathrm{E}-\mathrm{C}$ and the CesB synthetase shows the domain structure $\mathrm{A}_{\mathrm{i}}-\mathrm{X}-\mathrm{A}_{\mathrm{ii}}-\mathrm{T}-\mathrm{C}-\mathrm{A}-\mathrm{T}-\mathrm{TE}$ (Fig. 1). $A_{i}$ comprises the conserved core motifs $A 1-A 8$ while $A_{\mathrm{ii}}$ contains the core motifs $\mathrm{A} 9$ and $\mathrm{A} 10$, and $\mathrm{x}$ refers to a region of unknown function. Generally, the order of modules in a given NRPS correlates to the order of monomers in the peptide product, hence CesA is expected to activate the precursors for D-O-Leu (CesA1) and D-Ala (CesA2), while CesB is expected to be involved in the acti- vation of a precursor of L-O-Val (CesB1) and the activation and incorporation of the proteinogenic amino acid LVal (CesB2). Using the amino acid specificity code [26], the substrate activated by the modules CesA2 and CesB2 could be clearly predicted to be Ala and Val, while no A domain specificities could be predicted for the modules CesA1 and CesB1 (data not shown). However, alignment and genetic analysis of A domains revealed significant similarities between the latter modules, which are involved in the incorporation of the non-proteinogenic oxy acids (Fig. 2). A comparison of the A domain core motifs showed that these are less conserved in CesA 1 and CesB1 than in CesA2 and CesB2 (Fig. 3A). In addition, the A domains of CesA1 and CesB1 are interrupted by an insertion (x) of about 550 amino acids between the A8 and A9 core motifs. The inserted sequences from CesA1 and CesB1 showed 25\% identity and 40\% similarity. Comparison to database sequences revealed no significant homology of the first 320 aa of these sequences,

Table 2: Deduced function of encoded proteins in the ces operon and flanking regions

\begin{tabular}{|c|c|c|}
\hline Protein $^{\mathrm{a}}$ & Length of product (aa) & Deduced function (Homology) \\
\hline CDS I & 63 & Hypothetical protein ( $\mathrm{PXOI}$ related) \\
\hline CDS 2 & 159 & CAAX amino terminal protease \\
\hline CDS $\times 14$ & 565 & Conserved protein (Clp ATPase') (pXOI-I4 like) \\
\hline Ces gene cluster & & Cereulide biosynthesis \\
\hline $\mathrm{CesH}$ & 269 & Hydrolase/Acyltransferase \\
\hline CesP & 251 & Phosphopantheteinyltransferase \\
\hline CesT & 237 & Type II Thioesterase \\
\hline CesA & 3391 & NRPS (Cereulide synthesis) \\
\hline CesB & 2681 & NRPS (Cereulide synthesis) \\
\hline CesC & 291 & $A B C$ transporter \\
\hline CesD & 268 & $A B C$ transporter, Permease \\
\hline CDS $\times 11$ & 137 & Hypothetical protein (pXOI-II like) \\
\hline CDS $\times 23$ & 642 & Group II Intron (Reverse transcriptase, maturase) \\
\hline CDS $\times 10$ & 333 & Hypothetical protein (Methyltransferase $\left.{ }^{b}\right)(\mathrm{pXO}-10$ like) \\
\hline
\end{tabular}

a) CDS designation: CDSs with homolgies to $\mathrm{PXOI} C D S$ s are indicated by " $\mathrm{X}$ " and the corresponding CDS number of pXOI; CDSs belonging to the ces gene cluster are named ces; b) Putative function as determined by Ariel et al., [54]. 


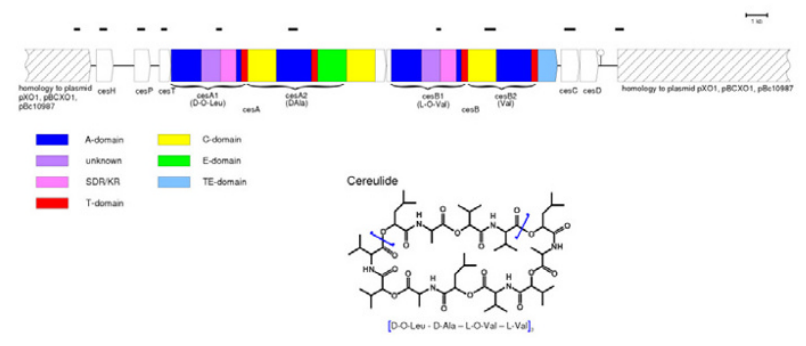

Figure I

Biosynthetic gene cluster for cereulide synthesis. The domain organization of the structural cereulide synthetase genes $\operatorname{ces} A$ and $\operatorname{ces} B$ is indicated (see Results for details) and the flanking regions showing homologies to toxin plasmids from $B$. cereus group members are printed as hatched boxes. For details on CDS designation see Table 2. The bars refer to probes used to test the conservation of ces genes in the $B$. cereus group (see Table 3 ) Inset: Structure of cereulide according to [13].

while the C-terminal part of the insertions showed significant similarities to enzymes belonging to the short chain dehydrogenase/reductase (SDR) superfamily (Fig. 3B). The module CesA2 contains an epimerase (E) domain, which is consistent with the D-stereochemistry of the Ala residue of cereulide. In the C-terminal region of $\operatorname{Ces} B$ a putative thioesterase (TE) domain comprising 254 amino acid residues (29 kDa) was identified (Table 2).

\section{Sequence and analysis of flanking regions of the ces operon}

The flanking regions of the ces operon were sequenced by inverse PCR and the resulting sequences were searched against NCBI's non-redundant database using BLAST algorithms. The 5 ' region and the 3 ' region of the ces genes showed significant homology (about $90 \%$ identity) to $B$. anthracis toxin-encoding pXO1 plasmid sequences and to pBc10987 [27]. To gain additional sequence information primers were designed using $B$. anthracis plasmid pXO1 sequence information. A total of $3.5 \mathrm{~kb}$ upstream and 9.5 $\mathrm{kb}$ downstream of the ces operon was sequenced. The corresponding upstream CDSs showed high identities $(60 \%$ to $98 \%$ ) to hypothetical proteins located on virulence plasmids of $B$. anthracis, $B$. thuringiensis, and B. cereus (pXO1 (GenBank Acc. no. AE017336), pBtoxis (GenBank Acc. no. AL731825), pE33L9 (GenBank Acc. no. CP000044), pBCXO1 (GenBank Acc. no. AAEK01000000) and pBc10987 (GenBank Acc. no. $\underline{\mathrm{AE} 017195)) .}$. Sequence analysis of the downstream region revealed 7 hypothetical proteins with homologies of more than $90 \%$ to hypothetical proteins from pXO1, pBCXO1 and $\mathrm{pBc} 10987$. The region downstream of the ces genes is nearly identical to the corresponding sequence of
pBc10987 (99\% identity), while no significant homology to pBtoxis could be observed. The sequence of the DNA region upstream from ces is $94 \%$ identical to the corresponding sequence of $\mathrm{pBc} 10987$ and $88 \%$ identical to pBtoxis,

respectively.

\section{PFGE and hybridization studies}

Undigested genomic DNA from selected emetic and emetic-like B. cereus strains was separated by PFGE, blotted on a membrane and hybridized to probes that were directed either against the cereulide synthetase genes (cesB probe obtained from emetic $B$. cereus) or against a CDS located in the flanking region of the ces genes (pXO1-11 probe obtained from $B$. anthracis). The hybridization results (see Fig. 4) showed that the cereulide synthetase is

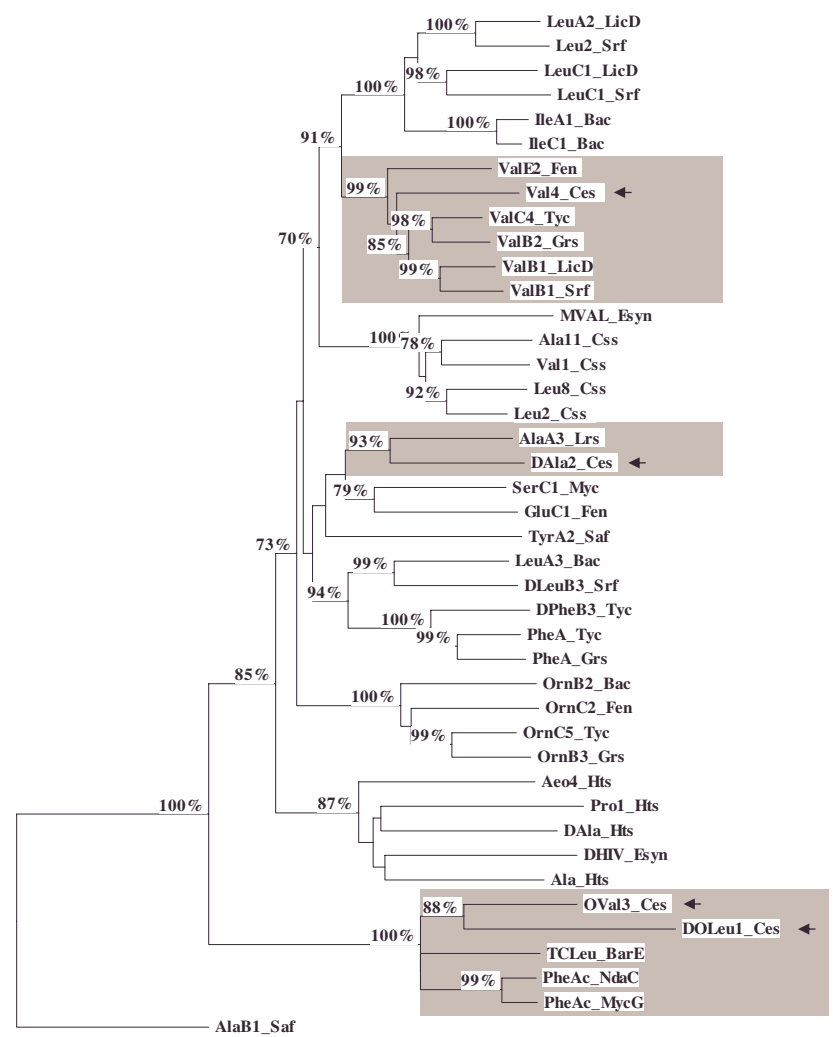

Figure 2

Genetic analysis of $\mathbf{A}$ domains. (A3 motif to the $A 6$ motif) from nonribosomal peptide synthetases. The tree was constructed with TREECON [48] using the neighbor-joining method. All bootstrap values $>70 \%$ ( 1000 replicates) are shown next to the nodes. A domains from the cereulide synthetase (Ces) are printed in bold type. Abbreviations: Bac: bacitracin synthetase; Bar: barbamide synthetase; Css: cyclosporine synthetase; Esyn: Enniatin; Fen: fengycin synthetase; Grs: gramicidin S synthetase; Hts: HC-toxin; LicD: lichenysin synthetase; Myc: mycosubtilin synthetase; Nda: nodularin synthetase; Saf: Saframycin synthetase; Srf: surfactin synthetase; Tyc: tyrocidine A synthetase. 
A

$\begin{array}{lllll} & \text { A4 } & \text { A5 } & \text { A6 } & \text { A7 } \\ \text { Consensus } & \text { FDxS } & \text { NXYGPTE } & \text { GELXIXGXG(VL)ARGY } & \text { Y(RK) TGDL } \\ \text { L-Val } & \text { FDAA } & \text { NGYGPTE } & \text { GELCVGGDGVAKGYV } & \text { YRTGDY } \\ \text { D-Ala } & \text { FDVS } & \text { NLYGPTE } & \text { GELCLGGICVAKGYM } & \text { YRTGDL } \\ \text { L-0-Val } & \text { HVGG } & \text { PVWGMSE } & \text { GRLQIKGKNVEGYY } & \text { YDTGDL } \\ \text { D-O-Leu } & \text { HVGG } & \text { PTFGMSE } & \text { GRFQIHGPTVMNGYF } & \text { FDSGDL } \\ \text { PksJ } & \text { HVGG } & \text { PAWGMSE } & \text { GRFQVSGLSVTSGYY } & \text { FETGDL } \\ \text { BarE } & \text { HVGG } & \text { PAFGMSE } & \text { GKLQVKGASVTSGYY } & \text { FRTGDL } \\ \text { MCYG-A } & \text { HVGA } & \text { PAFGMTE } & \text { GREQIQGNSVTKGYY } & \text { FTTDGL } \\ \text { NdCA } & \text { HVGA } & \text { PAFGMTE } & \text { GRLQIKGFSVTEGYY } & \text { FTTGDL } \\ \text { EntE } & \text { HNYA } & \text { QVFGMAE } & \text { GRLMTRGPYTFRGYY } & \text { YCSGDL } \\ \text { DhbE } & \text { HNYP } & \text { QVFGMAE } & \text { GHILTRGPYTIRGYY } & \text { YRTGDI } \\ & & & & \end{array}$

B

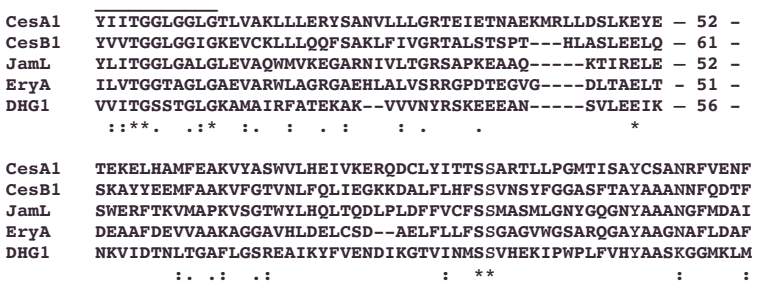

Figure 3

(A) Alignment of the adenylation. (A) domain core motifs lining the substrate binding pocket of Ces and unusual core motifs from other bacterial NRPS. Consensus sequence of core motifs A4 to A7 (according to [53]) is depicted. Residues identical to amino acids from Ces core motifs are printed in boldface type. (B) Insertions in A domains from CesA2 (D-O-Leu) and CesBI (L-O-Val) were aligned to short chain dehydrogenases (SDR) and ketoreductases $(K R)$; partial sequences including putative NADPH binding sites (solid bar) and the catalytic residues of SDRs/KR (printed in boldface type). Numbers between hyphens in the first lines refer to residues not shown before residues depicted in the second lines. JamL: KR from Jamaicamides synthetase (GenBank accession no. AY522504) of Lyngbya majuscula; EryA: KR from erythronolide synthetase of Saccharopolyspora erythraea (GenBank accession no. M63676).

GlcDh: Glucose I-dehydrogenase from B. megaterium (Swissprot accession no. P39482).

located on a megaplasmid, designated pBCE4810, of the same size as pBc10987 (208 kb). No hybridization signals were obtained from the non-emetic strains tested. Emeticlike strains reacted positively with the pXO1-11 derived probe but not with the ces probe. However, the sizes of the plasmids reacting with pXO1-11 differed slightly from that of the emetic strains (Fig. 4). Additional probes targeting different modules of the cereulide synthetase operon and its flanking regions were designed, and slot blot analysis was carried out to determine if parts of the ces gene locus are conserved in non-emetic $B$. cereus group strains. None of the non-emetic strains reacted with any of the ces genes derived probes (Table 3), while some of the strains, especially emetic-like strains, were positive in hybridization assays with pXO1 derived probes (Table 4). The corresponding plasmid from one of these emetic-like strains, pBCEL1519 from B. cereus NVH 1519-00, was partially sequenced using primers designed from pBCE4810 sequence with similarity to $\mathrm{pXO} 1$.

\section{Discussion}

\section{The cereulide synthetase operon}

We have sequenced a $36 \mathrm{~kb}$ genetic region in the $B$. cereus emetic reference strain F4810/72 involved in the biosynthesis of cereulide. Previous knock out mutants have shown that this toxin is produced by a multi-enzyme complex named cereulide synthetase [22]. The chemical structure of cereulide is reflected in the genetic organization of its synthetase genes (Fig. 1). This gene cluster comprises, besides the typical genes such as a 4'-phosphopantetheinyl transferase (cesP) essential for activating the NRPS (apoenzyme to holoenzyme) and the structural genes responsible for the assembly of the peptide product, a putative type II thioesterase (cesT) which could potentially remove misprimed monomers and regenerate the NRPS [28]. A putative hydrolase (cesH) is located in the 5' part of the cereulide biosynthesis cluster and a putative ABC transporter $(c e s C / D)$ is encoded in its 3' part. CesC/D might either be involved in the transport of cereulide or confer self resistance towards cereulide.

The structure of the ces genes and number of modules predicts a monomeric tetrapeptide but cereulide is a trimeric depsipeptide (Fig. 1). A similar structure has been described for gramicidin S and enterobactin. Synthesis of those peptides is carried out by one NRPS that is regenerated and repetitively used for synthesis. In case of such iterative processes the TE domains have additional functions allowing the collinear synthesis to be repeated two or three times (for review see [19]). The exact mechanism of these iterative TEs is not yet known, but mutation studies provided evidence that the TE domain of E. coli EntF catalyzes cyclolactonization as well as chain elongation [29]. A similar mechanism resulting in intramolecular trimerization might also be involved in cereulide assembly.

\section{Unusual structure of heterocompound activating modules}

The A domains of the heterocompound activating modules CesA1 and CesB1 are less conserved than the A domains from CesA2 and CesB2, which activate the amino acid moieties (Fig. 2). Especially, the core motifs A4 and A5 differ significantly from the typical consensus motifs described by Marahiel et al., [20]. Nevertheless, they show some homology to the corresponding motifs of phenylacetate activating modules $\mathrm{MycG}-\mathrm{A}$ and $\mathrm{NdaC}$ and to aryl acid activating modules DhbE and EntE [30,31] (see Fig. 3A). The crystal structures of the phenylalanineactivating A domain of gramicidin $S$ synthetase and the structure of DhbE involved in bacillibactin synthesis revealed a decisive role of the core motifs A4 and A5 in 


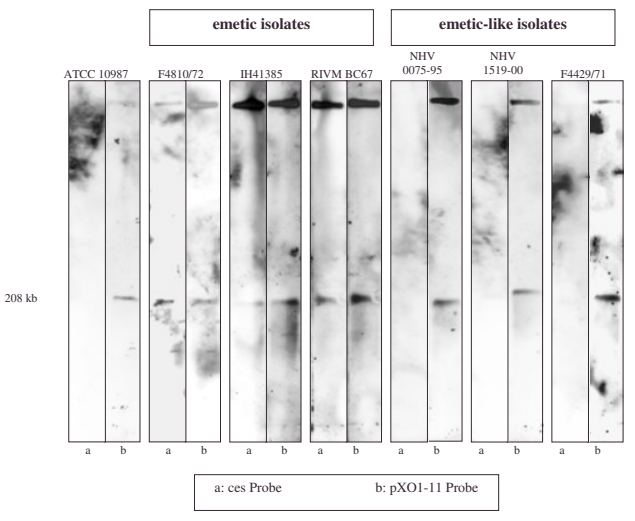

Figure 4

Hybridization of emetic and emetic-like $B$. cereus with probes targeting the ces operon (a) and a pXO I related CDS (b). Total DNA was separated by PFGE, transferred to a membrane and hybridized with a ces $B$ specific probe and a probe derived from $\mathrm{pXO}$-II (for details on probes see Supplemental Materials Table SI). Hybridization with both probes revealed a single band for emetic strains which has the same size as the pBcl0987 plasmid from B. cereus ATCC 10987 (lane 2).

substrate binding and selection [31,32]. The altered A4 and A5 core motifs found in CesA1 and CesB1 did not allow substrate prediction for these modules. The A4 motif usually represents the first anchor in amino acid activating domains, however this core motif is missing in DhbE as its substrate does not contain any $\alpha$-aminogroup. In DhbE the A4 motif, as well as the A5, is replaced by another sequence motif that is thought to be specific for carboxy acid activating enzymes [31] while the residue $\mathrm{K} 517$ is conserved in $\alpha$-amino acid activating domains, in the carboy activating domain of DhbE, as well as in CesA1 and CesB1 (data not shown). Since the A4 and A5 motifs of the two hydroxy acid incorporating modules of the cereulide synthetase are quite similar, it is tempting to speculate that the motifs are characteristic for a special type of substrate (see Fig. 3A). Detailed biochemical and structural analysis will be necessary to clarify the substrate specificity of these modified A domains. Nevertheless, the altered motifs found in CesA1, CesB1 and other NRPSs might be useful to identify yet unknown A domains activating new types of substrates.

In addition, about 550 amino acids are inserted between the core motifs A8 and A9 in CesA1 and CesB1 (Fig. 1). Different types of insertions in A domains located at this position have been described for bacterial NRPS such as methyltransferases and oxidoreductases [33,34]. Since the $\mathrm{N}$ terminal part of the insertion $(\mathrm{x})$ in CesA1 and CesB1 did not reveal any significant homologies to characterized proteins from database entries, no decisive function could be attributed to these domains in the cereulide synthetase. However, the $\mathrm{C}$ terminal part of both insertions showed homologies to short chain dehydrogenases (SDR) and ketoreductases (KR) (Fig. 3B). In addition to the Rossmann fold motif, SDR catalytic Tyr and Ser residues, which are also highly conserved in reductase domains from polyketide synthetases (KR domains) [35], were identified (Fig 3B). As in KR domains the catalytic Lys residue of SDRs is substituted by an Asn in CesA1 and CesB1. It is therefore tempting to speculate that the inserted domains $\mathrm{x}(\mathrm{KR})$ might indeed catalyze the ketoreduction of the substrates bound to CesA1 and CesB1, respectively.

\section{The megaplasmid encoded cereulide synthetase genes are} restricted to emetic strains

In order to investigate the distribution and conservation of the ces genes among B. cereus group members, Southern blot analysis was performed using different probes that target all CDSs belonging to the ces operon and hypothetical CDSs located in the periphery of these genes. Special emphasis was placed on emetic-like strains since these are closely related to emetic strains but do not produce cereulide [36]. Our results revealed a strict confinement of ces genes to cereulide producers (Table 3). The putative hydrolase encoded by ces $\mathrm{H}$ was not detected in the nonemetic $B$. cereus group strains and is therefore considered

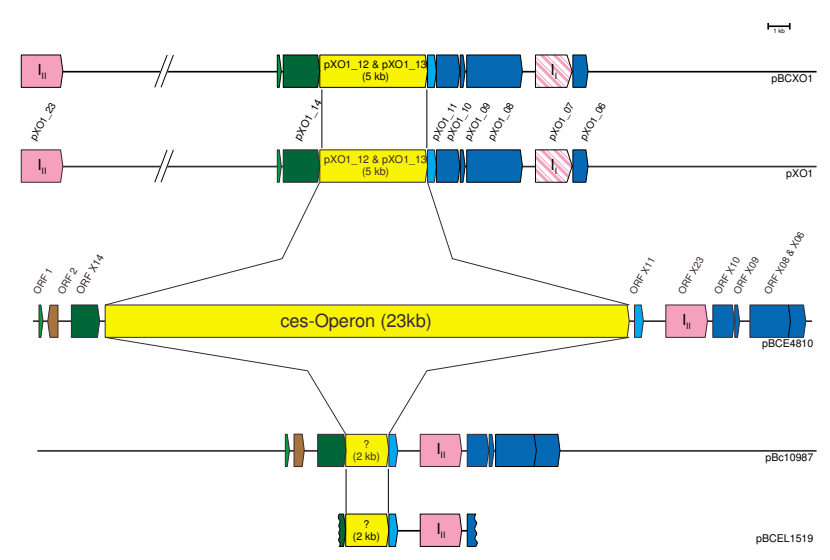

\section{Figure 5}

\section{Comparison of partial sequenced toxin plasmid} pBCE48 10 to toxin plasmids from $B$. cereus group members. The genetic region responsible for cereulide production in emetic strains was compared to the $B$. anthracis toxin-encoding plasmid $\mathrm{pXO}$, to $\mathrm{pBCXO}$ from $B$. cereus G924I capable of causing an anthrax-like illness, to pBcl0987 from B. cereus ATCC 10987 and to PBCELI5I9 from the emetic-like strain NVHI519-00. For CDS designation of pBCE and pBCEL see Table 2. $I_{1}$ : Group I intron; $I_{\|}$: Group II intron. 
Table 3: Occurrence of ces genes in B. cereus group members tested by hybridization assays. Several probes targeting all CDSs of the ces operon and CDSs from flanking regions (see Table 4) were used to test the distribution of ces genes in the $B$. cereus group.

\begin{tabular}{|c|c|c|c|c|c|c|c|c|c|}
\hline Strainb & Subtype $^{a}$ & cesH & cesP & cesT & cesAl & cesA2 & cesB I & cesB2 & $\operatorname{ces} \mathrm{C}$ \\
\hline \multicolumn{10}{|l|}{ B. cereus } \\
\hline F48I0/72 & $E$ & $x^{c}$ & $x$ & $x$ & $x$ & $x$ & $x$ & $x$ & $x$ \\
\hline MHI I305 & E & $x$ & $x$ & $x$ & $x$ & $x$ & $x$ & $x$ & $x$ \\
\hline UHDAM IH4I385 & $E$ & $x$ & $x$ & $x$ & $x$ & $x$ & $x$ & $x$ & $x$ \\
\hline NVH 0075-95 & EL & - & - & - & - & - & - & - & $(x)$ \\
\hline NVH I5I9-00 & EL & - & - & - & - & - & - & - & - \\
\hline INRA C24 & EL & - & - & - & - & - & - & - & - \\
\hline $\mathrm{F} 3003 / 73$ & $\mathrm{EL}$ & - & - & - & - & - & - & - & - \\
\hline F4429/7I & EL & - & - & - & - & - & - & - & - \\
\hline NVH 200 & EL & $(x)$ & - & - & - & - & - & - & - \\
\hline RIVM-BC-0063 & $\mathrm{EL}$ & - & - & - & - & - & - & - & - \\
\hline ATCC 10987 & EL & - & - & - & - & - & - & - & - \\
\hline WSBC 10892 & EL & - & - & - & - & - & - & - & - \\
\hline WSBC 10028 & NE & - & - & - & - & - & - & $(x)$ & $(x)$ \\
\hline WSBC 10035 & NE & - & - & - & - & - & - & - & - \\
\hline ATCC I4579' & NE & & & & & & & - & \\
\hline \multicolumn{10}{|l|}{ B. thuringiensis } \\
\hline WS 2620 & NE & - & - & - & - & - & - & - & - \\
\hline WS 2621 & NE & - & - & - & - & - & - & - & - \\
\hline WS 2632 & NE & - & - & - & - & - & - & - & - \\
\hline WSBC $2800 \mathrm{I}$ & NE & - & - & - & - & - & - & - & - \\
\hline WSBC 28002 & NE & - & - & - & - & - & - & - & - \\
\hline WSBC 28022 & NE & - & - & - & - & - & - & & - \\
\hline WSBC 28023 & NE & - & - & - & - & - & - & & - \\
\hline WSBC 28024 & NE & - & - & - & - & - & - & & - \\
\hline \multicolumn{10}{|l|}{ B. mycoides } \\
\hline WSBC 10256 & NE & - & - & - & - & - & - & - & - \\
\hline WSBC 10257 & NE & - & - & - & - & - & - & & - \\
\hline WSBC 10258 & NE & - & - & - & - & - & - & & - \\
\hline WSBC 10276 & NE & - & - & - & - & - & - & & - \\
\hline WSBC 10278 & NE & - & - & - & - & - & - & & - \\
\hline WSBC 10292 & NE & - & - & - & - & - & - & & - \\
\hline WSBC 10293 & NE & - & - & - & - & - & - & - & - \\
\hline WSBC 10360 & NE & - & - & - & - & - & - & - & - \\
\hline \multicolumn{10}{|l|}{ B. weihenstephanensis } \\
\hline WSBC 10001 & NE & - & - & - & - & - & - & & - \\
\hline WSBC 10045 & NE & - & - & - & - & - & - & & - \\
\hline WSBC 10202 & NE & - & - & - & - & - & - & & - \\
\hline WSBC $10204^{\top}$ & NE & - & - & $(x)$ & - & - & - & - & - \\
\hline WSBC 10212 & NE & - & - & - & - & - & - & & - \\
\hline WSBC 10296 & $\mathrm{NE}$ & - & - & - & - & - & - & & - \\
\hline
\end{tabular}

a) B. cereus group members are classified as emetic (E), emetic-like (EL) and non-emetic (NE) according to [36]. b) for strain designation and details on strains tested see additional file Table SI; c) X hybridization observed; $(X)$ weak hybridization signal; - no hybridization signal. 
an integral part of the ces gene locus. However, several non-emetic strains, especially emetic-like strains, were positive in hybridization studies using probes derived from ces flanking pXO1 homolog CDSs (Table 4). A high conservation of pXO1 genes among $B$. cereus group members and other closely related bacilli has previously been reported [37], but no information on emetic or emeticlike strains was provided in this study. Recently it has been reported that cereulide production depends on the presence of a plasmid. Cured emetic strains lost their ability to produce cereulide [38]. Our hybridization experiments (Fig. 4) showed that the cereulide synthetase encoding plasmid pBCE4810 is of the same size as the sequenced pXO1 related plasmid pBc10987 (208 kb) from B. cereus ATCC 10987 [27]. The latter, however, does not carry the ces genes. Plasmid sizes from emetic-like strains were slightly variable, but partial sequences of the plasmid pBCEL1519 (7.4 kb) from the emetic-like strain NVH1590-00 revealed an identity of nearly $100 \%$ to pBc10987 and 90\% to pXO1. A comparison of virulence plasmids from $B$. cereus group members and the partially sequenced plasmids pBCE4810 from the emetic B. cereus reference strain F4810/72, and pBCEL1519 from the emetic-like strain NVH1519-00, showed that the ces gene locus is inserted in a highly conserved part of these virulence plasmids between genes with similarities to pXO114 and pXO1-11 (Fig. 5). However, the inserts differ: at this location on pXO1 and pBCXO1 are the genes for
pXO1-13, a hypothetical virulence factor, and pXO1-12, whereas pBCE4810 contains the ces locus responsible for cereulide synthesis, and pBc10987 and pBCEL1519 both possess an identical $2 \mathrm{~kb}$ insert of unknown function. Downstream of this genetic locus a group II intron has recently been described in pBc10987 [39] that is also present in pBCE4810 and pBCEL1519 (Fig. 5). At the corresponding genetic region in $\mathrm{pXO} 1$ a group I intron has been identified [39]. Group I and group II introns were also found in other sequenced $B$. cereus group strains. Quite recently, evidence was provided that the group II introns splice in vivo [39]. This ability to splice is a prerequisite for mobility and insertion into new DNA target sites, and could become beneficial for the organism under stressful conditions.

\section{Evolution of different toxin plasmids from an ancient virulence plasmid in a B. cereus ancestor?}

The key role of the toxin plasmid pXO1 in anthrax pathogenesis and the importance of toxin harboring plasmids for insecticidal pathogenesis of $B$. thuringiensis are well known, whereas knowledge about the function of $B$. cereus plasmids is quite limited (for review see Rasko et al., [40]). Our present work revealed a third type of B. cereus group toxins being encoded by a megaplasmid: the biosynthetical genes responsible for the emetic type of $B$. cereus food borne disease are also located on a pXO1-like plasmid (Fig. 4). Thus, all species specific toxins of the $B$.

Table 4: Conservation of pXOI sequence in the emetic lineage of $B$. cereus.

\begin{tabular}{|c|c|c|c|c|c|c|c|c|c|c|c|}
\hline Strain & $\begin{array}{c}\text { Sub- } \\
\text { group }\end{array}$ & $\begin{array}{c}\text { pXOI- } \\
\text { II }\end{array}$ & $\begin{array}{c}\text { pXOI- } \\
14\end{array}$ & $\begin{array}{c}\text { pXOI- } \\
23\end{array}$ & $\begin{array}{c}\text { pXOI- } \\
55\end{array}$ & $\begin{array}{c}\text { pXOI- } \\
98\end{array}$ & $\begin{array}{c}\text { pXOI- } \\
\text { lef }\end{array}$ & $\begin{array}{c}\text { pXOI- } \\
\text { pagA }\end{array}$ & $\begin{array}{l}\text { pXOI- } \\
\text { atxA }\end{array}$ & $\begin{array}{c}\text { pXO I - } \\
\text { I36 } \\
\cot 43\end{array}$ & $\begin{array}{c}\text { pXOI- } \\
142\end{array}$ \\
\hline F48I0/72 & $\mathrm{E}$ & $x^{b}$ & $x$ & $x$ & $x$ & $x$ & - & - & - & $x$ & $x$ \\
\hline MHI I305 & E & $x$ & $x$ & $x$ & $x$ & $x$ & - & - & $(x)$ & $x$ & $x$ \\
\hline $\begin{array}{l}\text { UHDAM- } \\
\text { IH4I } 385\end{array}$ & $\mathrm{E}$ & $x$ & $x$ & $x$ & $x$ & - & - & - & - & $x$ & $x$ \\
\hline $\begin{array}{l}\text { NVH } \\
0075-95\end{array}$ & $E L$ & $x$ & $x$ & $x$ & $x$ & - & - & - & - & - & $x$ \\
\hline $\begin{array}{l}\text { NVH } \\
1519-00\end{array}$ & $\mathrm{EL}$ & $x$ & $x$ & $x$ & $x$ & $x$ & - & - & - & - & $x$ \\
\hline F4429/7I & $E L$ & - & $x$ & $x$ & $x$ & - & - & - & - & $(x)$ & - \\
\hline NVH 200 & $\mathrm{EL}$ & - & - & $x$ & $x$ & - & - & - & - & $x$ & $x$ \\
\hline $\begin{array}{l}\text { RIVM- } \\
\text { BC-0063 }\end{array}$ & EL & - & $x$ & $x$ & $(x)$ & $x$ & - & - & - & - & - \\
\hline $\begin{array}{l}\text { WSBC } \\
10892\end{array}$ & $E L$ & $x$ & - & $x$ & - & $x$ & - & - & - & $x$ & $x$ \\
\hline $\begin{array}{l}\text { WSBC } \\
10028\end{array}$ & NE & - & - & - & $(x)$ & - & - & - & - & - & $(x)$ \\
\hline $\begin{array}{l}\text { WSBC } \\
10035\end{array}$ & NE & - & $(x)$ & $x$ & $(x)$ & - & - & - & - & - & - \\
\hline $\begin{array}{l}\text { WSBC } \\
10204^{\top}\end{array}$ & NE & - & $(x)$ &.- & $x$ & - & - & - & - & - & - \\
\hline $\begin{array}{l}\text { ATCC } \\
\text { I } 4579 T^{T}\end{array}$ & NE & - & $x$ & - & $x$ & - & - & - & - & - & - \\
\hline
\end{tabular}

a) E: emetic strains and EL: emetic-like strains as defined by Ehling-Schulz et al., [36]; NE refers to non-emetic strains. b) $X$ hybridization observed; (X) weak hybridization signal; - no hybridization signal. 
cereus group are located on megaplasmids while the enterotoxins, which are broadly distributed among $B$. cereus group members [41], are localized on the chromosome. BLAST searches using the partial pBCE4810 sequence revealed high similarities (85-99\%) of the ces gene flanking regions to pXO1 and the $B$. cereus plasmids pBc10987 and the pXO1-like plasmid pBCXO1, and high similarities $(87-93 \%)$ in the 5 ' region of ces to the insecticidal toxin plasmid pBtoxis and a plasmid designated pE33L9 from a $B$. cereus strain isolated from a dead zebra. Comparison of selected pXO1-like CDSs from F4810/72 revealed an overall identity of about $95 \%$ of BCE4810 to pBc10987, about 92\% identity to pBCXO1, and about 90\% identity to pXO1. Like pBc10987, emetic strains seem to lack the $B$. anthracis PAI encoded virulence genes since no hybridization signals were obtained with probes targeting these genes (Table 4). The data presented in this work will contribute to developing a better understanding of the evolution and distribution of this group of plasmids. One might speculate that a plasmid in an ancestral form has been present in $B$. cereus group strains, which over time has acquired virulence genes conveying different disease causing phenotypes. Nevertheless, a detailed phylogenetic analysis of plasmids-focusing on mobile elements- from all members of the $B$. cereus group will be necessary to unravel the role of plasmids in pathogenesis and evolution of the B. cereus group of organisms.

\section{Conclusion}

The characterization of the ces genes illustrated the high flexibility of the module organization of bacterial NRPS. A new type of insertion in A domains has been observed in the modules CesA1 and CesB1. No clear function could be assigned to the inserted domains, although hundreds of NRPS modules have been sequenced and biochemically characterized. The characterization of the cereulide synthetase genes and its flanking regions revealed the extra-chromosomal location of the main virulence factor of emetic $B$. cereus on a plasmid with a pXO1-like backbone. Sequencing of the entire plasmids from emetic and emetic-like strains could provide new insight into the evolution of toxin producing members of the B. cereus group, especially elucidation of the evolution of $B$. anthracis and the emetic lineage of B. cereus.

\section{Methods}

\section{Bacterial strains}

The cereulide producing reference strain F4810/72 [42] was used to determine the sequence of the complete cereulide synthetase locus and the sequence of flanking regions. Cells were grown on plate count (PC) agar plates or in LB (Luria-Bertani) medium at $30^{\circ} \mathrm{C}$. Escherichia coli strains used for subcloning were grown at $37^{\circ} \mathrm{C}$ in $\mathrm{LB}$ medium with the appropriate antibiotics. Details on the origin of $B$. cereus group strains used to test the occurrence of the ces genes in hybridization studies are provided in Table S1 (see additional files).

\section{Sequencing strategies}

Due to stability problems with clones from a cosmid library, the sequence of the total cereulide synthetase operon and its flanking regions was determined by inverse PCR and module jumping as described previously [22]. The latter sequencing strategy took advantage of highly conserved core motifs in NRPS genes which have been successfully used to design degenerated primers and amplify novel NRPS modules [20,43]. With the combination of a specific primer located in the known sequence and a degenerated primer located in a core motif in a putative flanking module, additional sequence information can be gained by "jumping" from one module to the next one, see e.g. [22]. Organization of the ces gene cluster was confirmed by direct sequencing of the antisense strand from genomic DNA. In addition, primers derived from genomic sequences of the $B$. anthracis pXO1 toxin plasmid and the B. cereus ATCC 10987 pBc10987 plasmid were used to obtain sequence information from flanking regions of the cereulide synthetase operon. DNA was prepared using the AquaPure Genomic DNA Isolation-Kit (Bio-Rad, Germany). For inverse PCR total chromosomal DNA was isolated by phenol-chloroform extraction as described previously [43]. Preparation of plasmid DNA was performed according to standard procedures [44]. Sequences of degenerated primers successfully used for module jumping are listed in Table 1. Amplicons from module jumping were subcloned in TOPO TA vectors according to the manufacturer's recommendation (Invitrogen, USA) and sequenced as described previously [22]. PCR amplification products obtained with primers predicted from plasmids of $B$. cereus group members and PCR products obtained by inverse PCR were either subcloned in TOPO TA or directly sequenced by using a BigDye Terminator v1.1 Cycle Sequencing kit (Applied Biosystems).

\section{Sequence analysis}

The sequencing analysis software package Vector NTI (Informax Inc., U.S.A.) was used to generate the contig sequence from the sequenced PCR products obtained by PCR, inverse PCR and module jumping and the resulting sequence was searched against the sequenced genomes of $B$. cereus group members. Sequence similarity searches were performed using the Basic Local Aligment Search Tools BLASTX and BLASTP on the NCBI website $[45,46]$. The software packages ClustalX and TREECON were used for sequence alignments and cluster analysis $[47,48]$. Substrate specificity of ces genes was assessed according to Stachelhaus et al; [26]. The terminator search tool [49] available at Heidelberg Unix Sequence Analysis Resources [50] and Mfold [51], available at the Macfarlane Burnet 
Center's internet site [51], were used for the prediction of termination sequences.

\section{Hybridization assays}

For hybridization studies chromosomal DNA was isolated with the Puregene DNA isolation kit (Gentra, USA) according to the manufacturer's instruction and blotted onto nitrocellulose using a Milliblot-S slot blot manifold (Millipore, USA). Hybridization was performed using digoxigenin-labelled probes (Roche) directed against the different modules of ces, genes flanking the ces locus and selected pXO1 CDSs. The probes were obtained from the emetic reference strain F4810/72 and B. anthracis CIPA2 (provided by Gilles Vernaud, Orsay, France) and $B$. anthracis 6-87 (provided by Ulrich Busch, Oberschleissheim, Germany) by PCR using the oligonucleotide primers described in Table S2 (see additional files). Optimal hybridization temperature for each probe was calculated and hybridization was carried out according to recommendations of the manufacturer (Roche, Germany). After hybridization, membranes were washed twice for 15 min in $2 \times$ SSC containing $0.1 \%$ SDS at room temperature and two times $15 \mathrm{~min}$ in $0.1 \times$ SSC containing $0.1 \%$ SDS at $65^{\circ} \mathrm{C}$. Detection was performed by enzyme immunoassay according to recommendation of manufacture (Roche, Germany) with the chemiluminescence CDP-Star AP substrate (Novagen, USA).

\section{Pulse field gel electrophoresis (PFGE)}

Preparation of total genomic DNA in agarose plugs was performed as described by Kolsto et al., [52]. Electrophoresis was performed using a CHEF DR III System (BioRad) with a $0.8 \%$ SeaKem Gold Agarose gel (Cambrex Bio Science, USA) in $0.25 \times$ TBE buffer $(25 \mathrm{mM}$ Tris-borate buffer $\mathrm{pH} 8,0.05 \mathrm{mM}$ EDTA) at $15^{\circ} \mathrm{C}$ with a pulse of 5$200 \mathrm{~s}$ for 20 hours. Size of the fragments was estimated using lambda concatamers (Bio-Rad) and Salmonella Braenderup Global Standard (PulseNet) H9812 (XbaI digested). After electrophoresis, gels were stained in 100 $\mu \mathrm{g} / \mathrm{ml}$ ethidium bromide for $30 \mathrm{~min}$ and destained in water for $1 \mathrm{~h}$. Gels were denaturated in $0.25 \mathrm{M} \mathrm{HCl}$ for 15 min, followed by soaking the gel in $1.5 \mathrm{M} \mathrm{NaCl} / 0.5 \mathrm{~N}$ $\mathrm{NaOH}$ with constant agitation for $2 \times 30 \mathrm{~min}$. Neutralization was performed in $0.5 \mathrm{M}$ Tris $(\mathrm{pH} 8) / 1.5 \mathrm{M} \mathrm{NaCl}$ for $2 \times 30 \mathrm{~min}$. Gels were blotted overnight by capillary transfer in $20 \times$ SSC onto Hybond-N+ nitrocellulose membrane (Amersham Biosciences, UK). After blotting, the membranes were rinsed in $6 \times \mathrm{SSC}$, air dried for $30 \mathrm{~min}$ at room temperature and finally baked for $2 \mathrm{~h}$ at $80^{\circ} \mathrm{C}$. Hybridization was performed as described above.

\section{Nucleotide sequence accession number}

The nucleotide sequence from pBCE4810 of B. cereus F4810/72 described in this paper has been submitted to GenBank under accession no. DQ360825.

\section{Authors' contributions}

MES was in charge of sequencing and data analysis, conceived the study and did the writing. MF was in charge of the hybridization studies, did part of the sequencing and participated in writing the method section. HG worked at the project as diploma student, doing part of the sequencing and gene cluster analysis. PR and MW performed the PFGE experiments. SS supported the study, participated in its design and contributed to writing.

\section{Additional material}

\section{Additional File 1}

Table S1: Origin of B. cereus group strains used for hybridization studies Click here for file

[http://www.biomedcentral.com/content/supplementary/14712180-6-20-S1.doc]

\section{Additional File 2}

Table S2: Oligonucleotide primers used for southern hybridization and sequencing

Click here for file

[http://www.biomedcentral.com/content/supplementary/14712180-6-20-S2.xls]

\section{Acknowledgements}

This work was supported by the European Commission (QLKI-CT-200I00854). We thank Kathrin Buntin and Sebastian Auer for excellent technical assistance and Monica Dommel for proofreading the manuscript.

\section{References}

I. Turnbull PC: Introduction: anthrax history, disease and ecology. Curr Top Microbiol Immunol 2002, 27 I: I- I9.

2. Mock M, Fouet A: Anthrax. Annu Rev Microbiol 200I, 55:647-67I.

3. Aronson $\mathrm{Al}$, Shai $\mathrm{Y}$ : Why Bacillus thuringiensis insecticidal toxins are so effective: unique features of their mode of action. FEMS Microbiol Lett 2001, I95(I): I-8.

4. Granum PE: Bacillus cereus. In Food Microbiology: Fundamentals and Frontiers 2nd edition. Edited by: Doyle MP. Washington D.C. , ASM Press; 2001:373-381.

5. Ehling-Schulz M, Fricker M, Scherer S: Bacillus cereus, the causative agent of an emetic type of food-borne illness. Mol Nutr Food Res 2004, 48(7):479-487.

6. Granum PE: Bacillus cereus and its toxins. Journal of Applied Bacteriology Symposium Supplement 1994, 76:6I S-66S.

7. Beecher DJ, Wong AC: Tripartite haemolysin BL: isolation and characterization of two distinct homologous sets of components from a single Bacillus cereus isolate. Microbiology 2000 , | 46 ( Pt 6): | 37| - |380.

8. Lindback T, Fagerlund A, Rodland MS, Granum PE: Characterization of the Bacillus cereus Nhe enterotoxin. Microbiology 2004 , I 50(Pt I 2):3959-3967.

9. Lund T, De Buyser ML, Granum PE: A new cytotoxin from Bacillus cereus that may cause necrotic enteritis. Mol Microbio 2000, 38(2):254-26I.

10. Brillard J, Lereclus D: Comparison of cytotoxin cytK promoters from Bacillus cereus strain ATCC 14579 and from a B. cereus food-poisoning strain. Microbiology 2004, I 50(8):2699-2705

II. Dierick K, Van Coillie E, Swiecicka I, Meyfroidt G, Devlieger H, Meulemans A, Hoedemaekers G, Fourie L, Heyndrickx M, Mahillon J: Fatal family outbreak of Bacillus cereus-associated food poisoning. I Clin Microbiol 2005, 43(8):4277-4279. 
12. Mahler H, Pasi A, Kramer JM, Schulte P, Scoging AC, Bar W, Krahenbuhl S: Fulminant liver failure in association with the emetic toxin of Bacillus cereus. N Engl J Med I997, 336( I6): | |42-I | 48.

13. Agata N, Mori M, Ohta M, Suwan S, Ohtani I, Isobe M: A nove dodecadepsipeptide, cereulide, isolated from Bacillus cereus causes vacuole formation in HEp-2 cells. FEMS Microbiol Lett | 994, I 2 I ( I):3 |-34

14. Paananen A, Mikkola R, Sareneva T, Matikainen S, Hess M, Andersson M, Julkunen I, Salkinoja-Salonen MS, Timonen T: Inhibition of human natural killer cell activity by cereulide, an emetic toxin from Bacillus cereus. Clin Exp Immunol 2002, I 29(3):420-428.

15. Turgay K, Krause M, Marahiel MA: Four homologous domains in the primary structure of GrsB are related to domains in a superfamily of adenylate-forming enzymes. Mol Microbiol 1992 , 6(I 8):2743-2744.

16. Konz D, Marahiel MA: How do peptide synthetases generate structural diversity? Chem Biol 1999, 6(2):R39-48.

17. Stachelhaus T, Mootz HD, Bergendahl V, Marahiel MA: Peptide bond formation in nonribosomal peptide biosynthesis. Catalytic role of the condensation domain. J Biol Chem 1998 273(35):22773-2278I.

18. Trauger JW, Kohli RM, Mootz HD, Marahiel MA, Walsh CT: Peptide cyclization catalysed by the thioesterase domain of tyrocidine synthetase. Nature 2000, 407(680I):215-218.

19. Sieber SA, Marahiel MA: Molecular mechanisms underlying nonribosomal peptide synthesis: approaches to new antibiotics. Chem Rev 2005, I05(2):715-738.

20. Marahiel MA, Stachelhaus T, Mootz HD: Modular peptide synthetases involved in nonribosomal peptide synthesis. Chem $\operatorname{Rev} 1997,97(7): 2651-2673$

21. von Döhren H, Keller U, Vater J, Zocher R: Multifunctional peptide synthetases. Chemical Reviews 1997, 97:2675-2705.

22. Ehling-Schulz M, Vukov N, Schulz A, Shaheen R, Andersson M, Martlbauer E, Scherer S: Identification and partial characterization of the nonribosomal peptide synthetase gene responsible for cereulide production in emetic Bacillus cereus. Appl Environ Microbiol 2005, 7 I (I): I05-II3.

23. Zuker M: Mfold web server for nucleic acid folding and hybridization prediction. Nucleic Acids Res 2003, 3 I (I3):3406-34 I5.

24. Quadri LE, Weinreb PH, Lei M, Nakano MM, Zuber P, Walsh CT: Characterization of Sfp, a Bacillus subtilis phosphopantetheinyl transferase for peptidyl carrier protein domains in peptide synthetases. Biochemistry 1998, 37(6): I 585-I595.

25. Borchert S, Stachelhaus T, Marahiel MA: Induction of surfactin production in Bacillus subtilis by gsp, a gene located upstream of the gramicidin $\mathbf{S}$ operon in Bacillus brevis. J Bacteriol 1994, I 76(8):2458-2462.

26. Stachelhaus T, Mootz HD, Marahiel MA: The specificity-conferring code of adenylation domains in nonribosomal peptide synthetases. Chem Biol 1999, 6(8):493-505.

27. Rasko DA, Ravel J, Okstad OA, Helgason E, Cer RZ, Jiang L, Shores KA, Fouts DE, Tourasse NJ, Angiuoli SV, Kolonay J, Nelson WC, Kolsto $A B$, Fraser CM, Read TD: The genome sequence of Bacillus cereus ATCC 10987 reveals metabolic adaptations and a large plasmid related to Bacillus anthracis pXO I. Nucleic Acids Research 2004, 32(3):977-988.

28. Schwarzer D, Mootz HD, Linne U, Marahiel MA: Regeneration of misprimed nonribosomal peptide synthetases by type II thioesterases. Proc Natl Acad Sci U S A 2002, 99(22): |4083-| 4088.

29. Shaw-Reid CA, Kelleher NL, Losey HC, Gehring AM, Berg C, Walsh CT: Assembly line enzymology by multimodular nonribosomal peptide synthetases: the thioesterase domain of E. coli EntF catalyzes both elongation and cyclolactonization. Chem Biol 1999, 6(6):385-400.

30. Moffitt MC, Neilan BA: Characterization of the nodularin synthetase gene cluster and proposed theory of the evolution of cyanobacterial hepatotoxins. Appl Environ Microbiol 2004, 70(I I):6353-6362.

31. May |J, Kessler N, Marahiel MA, Stubbs MT: Crystal structure of DhbE, an archetype for aryl acid activating domains of modular nonribosomal peptide synthetases. Proc Natl Acad Sci U S A 2002, 99( I 9): | 2 | 20-12125.

32. Conti E, Stachelhaus T, Marahiel MA, Brick P: Structural basis for the activation of phenylalanine in the non- ribosomal biosynthesis of gramicidin S. Embo J 1997, I 6(14):4174-4183.
33. Haese A, Schubert M, Herrmann M, Zocher R: Molecular characterization of the enniatin synthetase gene encoding a multifunctional enzyme catalysing N-methyldepsipeptide formation in Fusarium scirpi. Mol Microbiol I993, 7(6):905-9|4.

34. Walsh CT, Chen H, Keating TA, Hubbard BK, Losey HC, Luo L, Marshall CG, Miller DA, Patel HM: Tailoring enzymes that modify nonribosomal peptides during and after chain elongation on NRPS assembly lines. Curr Opin Chem Biol 200I, 5(5):525-534.

35. Reid R, Piagentini M, Rodriguez E, Ashley G, Viswanathan N, Carney J, Santi DV, Hutchinson CR, McDaniel R: A model of structure and catalysis for ketoreductase domains in modular polyketide synthases. Biochemistry 2003, 42(I):72-79.

36. Ehling-Schulz M, Svensson B, Guinebretiere $M H$, Lindback T, Andersson M, Schulz A, Fricker M, Christiansson A, Granum PE, Martlbauer E, Nguyen-The C, Salkinoja-Salonen M, Scherer S: Emetic toxin formation of Bacillus cereus is restricted to a single evolutionary lineage of closely related strains. Microbiology 2005, I5 I(I): I83-197.

37. Pannucci J, Okinaka RT, Sabin R, Kuske CR: Bacillus anthracis pXOI plasmid sequence conservation among closely related bacterial species. Journal of Bacteriology 2002, I 84(I): | 34-|4|

38. Hoton FM, Andrup L, Swiecicka I, Mahillon J: The cereulide genetic determinants of emetic Bacillus cereus are plasmid-borne. Microbiology 2005, I 5 I:2 I 21-2124.

39. Tourasse NJ, Stabell FB, Reiter L, Kolsto AB: Unusual group II introns in bacteria of the Bacillus cereus group. I Bacteriol 2005, I 87( I 5):5437-545।

40. Rasko DA, Altherr MR, Han CS, Ravel J: Genomics of the Bacillus cereus group of organisms. FEMS Microbiology Reviews 2005, 29(2):303-329.

4l. Pruss BM, Dietrich R, Nibler B, Martlbauer E, Scherer S: The hemolytic enterotoxin HBL is broadly distributed among species of the Bacillus cereus group. Appl Environ Microbiol 1999, 65(I 2):5436-5442

42. Häggblom MM, Apetroaie C, Andersson MA, Salkinoja-Salonen MS Quantitative analysis of cereulide, the emetic toxin of Bacillus cereus, produced under various conditions. Appl Environ Microbiol 2002, 68(5):2479-2483

43. Ehling-Schulz M, Fricker M, Scherer S: Identification of emetic toxin producing Bacillus cereus strains by a novel molecular assay. FEMS Microbiology Letters 2004, 232:189-195.

44. Sambrook J, Fritsch EF, Maniatis T: Molecular cloning: a laboratory manual. 2nd ed. edition. Cold Spring Harbor, N. Y. , Cold Spring Harbor Laboratory Press; 1989.

45. Altschul SF, Gish W, Miller W, Myers EW, Lipman DJ: Basic local alignment search tool. J Mol Biol 1990, 2I 5(3):403-4I0.

46. Altschul SF, Madden TL, Schaffer AA, Zhang J, Zhang Z, Miller W, Lipman DJ: Gapped BLAST and PSI-BLAST: a new generation of protein database search programs. Nucleic Acids Res 1997, 25( I 7):3389-3402.

47. Thompson JD, Gibson TJ, Plewniak F, Jeanmougin F, Higgins DG: The CLUSTAL_ $X$ windows interface: flexible strategies for multiple sequence alignment aided by quality analysis tools. Nucleic Acids Res 1997, 25(24):4876-4882.

48. Van de Peer Y, De Wachter R: Construction of evolutionary distance trees with TREECON for Windows: accounting for variation in nucleotide substitution rate among sites. Comput Appl Biosci 1997, I3(3):227-230.

49. Brendel $V$, Trifonov EN: A computer algorithm for testing potential prokaryotic terminators. Nucleic Acids Res 1984, | 2( I 0):44 | |-4427.

50. HUSAR http://geniusembnetdkfz-heidelbergde [http://gen ius.embnet.dkfz-heidelberg.de].

51. Mfold [http://mfold.burnet.edu.au]

52. Kolsto $A B$, Gronstad A, Oppegaard H: Physical map of the Bacillus cereus chromosome. J Bacteriol 1990, I 72(7):382 I-3825.

53. Marahiel MA: Protein templates for the biosynthesis of peptide antibiotics. Chem Biol 1997, 4(8):56I-567.

54. Ariel N, Zvi A, Grosfeld H, Gat O, Inbar Y, Velan B, Cohen S, Shafferman $A$ : Search for potential vaccine candidate open reading frames in the Bacillus anthracis virulence plasmid $\mathrm{pXO}$ : in silico and in vitro screening. Infect Immun 2002, 70(1 2):6817-6827. 